\title{
Distribution of Persistent Currents in a Multi-Arm Mesoscopic Ring
}

\author{
Santanu K. Maiti, ${ }^{1,2}$ Srilekha Saha, ${ }^{1}$ and S. N. Karmakar ${ }^{1}$ \\ ${ }^{1}$ Theoretical Condensed Matter Physics Division, Saha Institute of Nuclear Physics, \\ Sector-I, Block-AF, Bidhannagar, Kolkata-700 064, India \\ ${ }^{2}$ Department of Physics, Narasinha Dutt College, 129 Belilious Road, Howrah-711 101, India
}

\begin{abstract}
We propose an idea to investigate persistent current in individual arms of a multi-arm mesoscopic ring. Following a brief description of persistent current in a traditional Aharonov-Bohm (AB) ring, we examine the behavior of persistent currents in separate arms of a three-arm mesoscopic ring. Our analysis may be helpful in studying magnetic response of any complicated quantum network.
\end{abstract}

PACS numbers: 73.23.-b, 73.23.Ra.

\section{INTRODUCTION}

Generation of persistent current in a normal metal mesoscopic ring threaded by an AB flux $\phi$ has been proposed over a number of decades. The appearance of discrete energy levels and large phase coherence length allow a non-decaying current in presence of an AB flux $\phi$. Following the pioneering work of Büttiker et al. 11], various efforts have been made to explore the basic mechanisms of persistent current in mesoscopic rings and cylinders [210]. Later, the existence of non-decaying current in these systems has also been verified through some nice experiments 11 14. The behavior of persistent current in a mesoscopic ring/cylinder can be studied theoretically by several ways as available in the literature [1-10]. In all these available procedures response of the entire system is achieved only, but no information about individual branches of the system can be explored though it is highly significant to elucidate the actual mechanism of electron transport in a more transparent way. This is the main motivation behind this work.

With a brief description of persistent current in a single-channel mesoscopic ring, we discuss elaborately the behavior of persistent currents in individual arms of a three-arm mesoscopic ring. In this three-arm ring system we address an unconventional feature of persistent current when impurities are introduced only in the middle arm, keeping the other two arms free from any impurity. It shows that the current amplitude of the system increases with the increase of impurity strength in the strong impurity regime, while it decreases in the weak impurity regime. This phenomenon is completely different from traditional disordered systems, and recently, few anomalous features of electron dynamics have also been reported in some other nano materials [15, 16].

In what follows, we present the results. Section II is devoted to present the theory. The results are discussed in Section III. We provide a summary in Section IV.

\section{THEORETICAL FORMULATION}

A simple ring: Let us start with Fig. 1] where a mesoscopic ring is subject to an $\mathrm{AB}$ flux $\phi$ (measured in unit of the elementary flux quantum $\left.\phi_{0}=c h / e\right)$. A tightbinding (TB) formalism is given for the description of the ring. Within the non-interacting picture the TB Hamiltonian for a $N$-site ring looks in the form,

$$
\boldsymbol{H}=\sum_{i} \epsilon_{i} c_{i}^{\dagger} c_{i}+\sum_{i} v\left(c_{i+1}^{\dagger} c_{i} e^{j \theta}+c_{i}^{\dagger} c_{i+1} e^{-j \theta}\right)
$$

where, $\epsilon_{i}$ is the site energy and $v$ gives the nearestneighbor hopping integral. Due to the presence of $\mathrm{AB}$ flux $\phi$, a phase factor $\theta=2 \pi \phi / N$ appears in the Hamil-

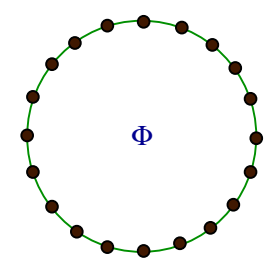

FIG. 1: A mesoscopic ring threaded by an AB flux $\phi$.

tonian. $c_{i}^{\dagger}\left(c_{i}\right)$ is the creation (annihilation) operator.

The energy $E_{k}$ corresponding to $k$-th energy eigenstate $\left|\psi_{k}\right\rangle$ can be obtained from the relation $E_{k}=\left\langle\psi_{k}|\boldsymbol{H}| \psi_{k}\right\rangle$, where $\left|\psi_{k}\right\rangle=\sum_{p} a_{p}|p\rangle$. Here $|p\rangle$ 's are the Wannier states and $a_{p}$ 's are the coefficients. Simplifying the above relation we get the expression of the energy $E_{k}$ as,

$$
E_{k}=\sum_{i} \epsilon_{i} a_{i}^{*} a_{i}+\sum_{i} v\left[a_{i+1}^{*} a_{i} e^{j \theta}+a_{i}^{*} a_{i+1} e^{-j \theta}\right]
$$

where, $a_{i}^{*}$ is the complex conjugate of $a_{i}$. Here, the summation index $i$ runs over all the atomic sites in the ring.

Now, the current carried by the $k$-th energy eigenstate $\left|\psi_{k}\right\rangle$ can be determined from the relation,

$$
I_{k}=-\frac{\partial E_{k}}{\partial \phi}=\frac{2 \pi j v}{N} \sum_{i}\left[a_{i}^{*} a_{i+1} e^{-j \theta}-a_{i+1}^{*} a_{i} e^{j \theta}\right] .
$$

At absolute zero temperature $(T=0 \mathrm{~K})$, the net persistent current for a ring described with $N_{e}$ electrons can be determined by taking the sum of individual contributions from the lowest $N_{e}$ energy eigenstates. Therefore, for $N_{e}$ electron system the net current becomes $I=\sum_{k=1}^{N_{e}} I_{k}$. 
A three-arm ring: Following the above prescription, the distribution of persistent currents in individual arms, viz, upper, middle and lower, of a three-arm mesoscopic ring shown in Fig. 2 can be established.

The TB Hamiltonian for this system is in the form,

$$
\begin{aligned}
\boldsymbol{H} & =\sum_{i} \epsilon_{i} c_{i}^{\dagger} c_{i}+\sum_{i} v_{1}\left(c_{i+1}^{\dagger} c_{i} e^{j \theta_{1}}+c_{i}^{\dagger} c_{i+1} e^{-j \theta_{1}}\right) \\
& +\sum_{l} \epsilon_{l} c_{l}^{\dagger} c_{l}+\sum_{l} v_{2}\left(c_{l+1}^{\dagger} c_{l} e^{j \theta_{2}}+c_{l}^{\dagger} c_{l+1} e^{-j \theta_{2}}\right)(4)
\end{aligned}
$$

where, the summation index $i$ is used to refer the atomic sites in the upper and lower arms of the ring i.e., in the outer ring, while the index $l$ describes the atomic sites in the middle arm (filled black circles in the framed region). $v_{1}$ and $v_{2}$ describe the nearest-neighbor hopping integrals in the outer ring and middle arm, respectively.

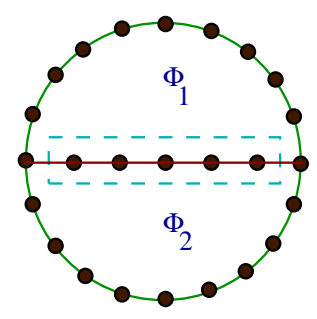

FIG. 2: A three-arm ring, where the upper and lower subrings are subject to $\mathrm{AB}$ fluxes $\phi_{1}$ and $\phi_{2}$, respectively.

The phase factor $\theta_{1}$ is associated with the hopping of an electron in the upper/lower arm, while in the middle arm it is described by the term $\theta_{2}$. They are represented as, $\theta_{1}=\left(\phi_{1}+\phi_{2}\right) /\left(N_{U}+N_{L}\right)$ and $\theta_{2}=\left(\phi_{1}-\phi_{2}\right) / 2 N_{M}$, where $N_{U}, N_{M}$ and $N_{L}$ represent the total number of single bonds (a bond is formed by connecting two neighboring atoms through a line) in the upper, middle and lower arms of the ring, respectively. For this geometry $E_{k}$ can be calculated according to the same prescription as mentioned above, and, with this energy expression we can determine the currents in individual arms. The expressions for the currents are as follows.

For the upper arm:

$$
I_{k}^{U}=\frac{2 \pi j v_{1}}{N_{U}+N_{L}} \sum_{i}\left[a_{i}^{*} a_{i+1} e^{-j \theta_{1}}-a_{i+1}^{*} a_{i} e^{j \theta_{1}}\right]
$$

where, contributions from the $N_{U}$ bonds are added.

For the middle arm:

$$
I_{k}^{M}=\frac{\pi j v_{2}}{N_{M}} \sum_{l}\left[a_{l}^{*} a_{l+1} e^{-j \theta_{2}}-a_{l+1}^{*} a_{l} e^{j \theta_{2}}\right]
$$

here, net contribution comes from $N_{M}$ bonds.

For the lower arm:

$$
I_{k}^{L}=\frac{2 \pi j v_{1}}{N_{U}+N_{L}} \sum_{i}\left[a_{i}^{*} a_{i+1} e^{-j \theta_{1}}-a_{i+1}^{*} a_{i} e^{j \theta_{1}}\right]
$$

here, the individual contributions of $N_{L}$ bonds are added. Using these relations (Eqs. 5[7]), the net persistent currents at $T=0 \mathrm{~K}$ in individual arms of a three-arm mesoscopic ring containing $N_{e}$ electrons can be obtained in the same fashion as mentioned earlier.

In the present work we perform all the characteristics of persistent current at $T=0 \mathrm{~K}$ and use the units where $c=h=e=1$. Throughout our numerical work we set the nearest-neighbor hopping strengths $\left(v, v_{1}\right.$ and $\left.v_{2}\right)$ to -1 and the energy scale is measured in unit of $v$.

\section{NUMERICAL RESULTS AND DISCUSSION}

A simple ring: As illustrative examples in Fig. 3 we show the current-flux characteristics for a single-channel impurity-free $\left(\epsilon_{i}=0\right.$ for all $\left.i\right)$ mesoscopic ring considering $N=20$, where (a) and (b) correspond to the results for odd and even number of electrons, respectively. Both for the cases of odd and even $N_{e}$, current provides sawtooth like nature with sharp jumps at half-integer or integer multiples of flux-quantum $\phi_{0}$ depending on whether
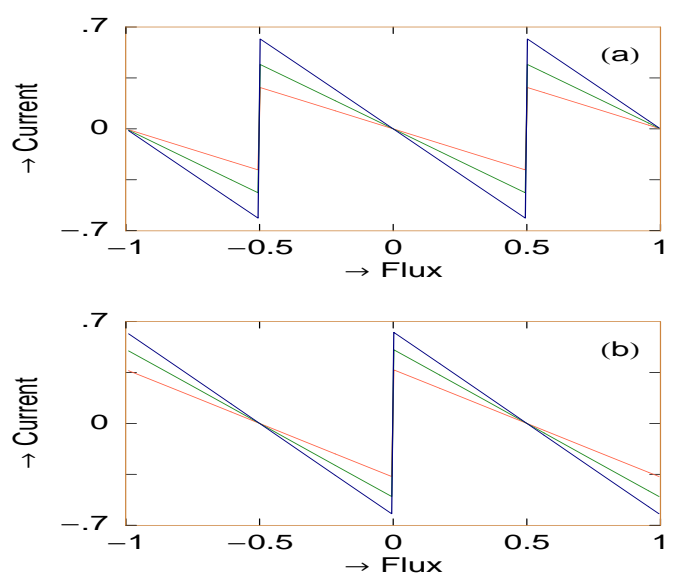

FIG. 3: $I-\phi$ spectra of an ordered mesoscopic ring $(N=20)$ with (a) odd $N_{e}$ and (b) even $N_{e}$. The red, green and blue curves in (a) correspond to $N_{e}=3,5$ and 9, respectively, while in (b) they represent $N_{e}=4,6$ and 10, respectively.

the ring is described by odd or even number of electrons. These currents are periodic in $\phi$ exhibiting $\phi_{0}$ fluxquantum periodicity. The presented current-flux characteristics for this single-channel ring exactly match with the previous theoretical studies where persistent currents have been calculated by other approaches [1 10].

Thus, making sure with the results for a single-channel ring now we can safely use this procedure to illustrate the current-flux characteristics in individual arms of a threearm mesoscopic ring.

\section{A three-arm ring:}

Ring without any impurity: In Fig. 4 we present currentflux characteristics for an ordered $\left(\epsilon_{i}=\epsilon_{l}=0\right.$ for all $i$ and $l$ ) three-arm mesoscopic ring, keeping $\phi_{2}$ constant. 
These currents are evaluated for a fixed number of electrons $N_{e}=10$ and they provide a complex spectra. It is noticed that the responses of the individual branches are quite different from each other, especially, a significant change in amplitude takes place among the currents $I_{U}, I_{M}$ and $I_{L}$. By adding these three currents we get the net current $\left(I_{T}\right)$ for the entire ring and it becomes exactly identical to the current determined from the other conventional methods available in the literature [1-10]. This emphasizes the current conservation relation $I_{T}=I_{U}+I_{M}+I_{L}$. Other important feature
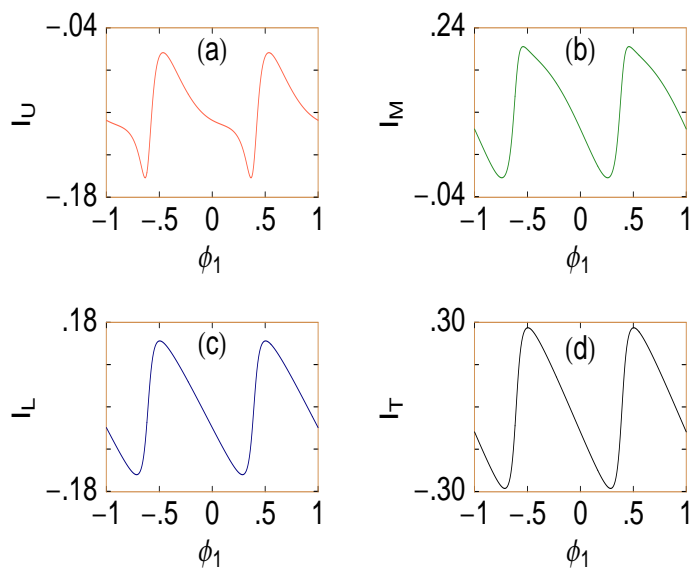

FIG. 4: Persistent current as a function of $\phi_{1}$ for an ordered three-arm mesoscopic ring considering $N_{U}=N_{L}=10, N_{M}=$ $9, \phi_{2}=0.3$ and $N_{e}=10$. (a), (b) and (c) correspond to the currents in the upper, middle and lower arms, respectively, while (d) gives the total current for the entire three-arm ring.

is that the sum of the currents carried by the upper and middle arms becomes exactly identical to the current carried by the lower arm i.e., $I_{L}=I_{U}+I_{M}$ which gives another relation of current conservation. All these currents exhibit $\phi_{0}$ flux-quantum periodicity.

Now, instead of $\phi_{1}$ if we plot the currents as a function of $\phi_{2}$, considering $\phi_{1}$ constant, exactly similar features are observed, like above, satisfying the current conservation relations.

Ring with impurities in the middle arm: To introduce impurities in the middle arm we choose the site energies of the atomic sites, filled black circles in the framed region of Fig. 2, randomly from a "Box" distribution function of width $W$. Here, we determine the currents by taking the average over 50 random disordered configuration in each case to achieve much accurate results.

As the middle arm is subject to impurities, while the others are free, we call this system as an ordereddisordered separated three-arm mesoscopic ring. In such a mesoscopic ring an unconventional feature of persistent current is observed when we tune the strength of disorder $W$. To emphasize it, in Fig. 5 we show the variation of persistent current in individual arms for a threearm mesoscopic ring considering $N_{U}=N_{L}=15$ and
$N_{M}=11$. From the spectra it is observed that when $W=1$ (weak), the current amplitude gets reduced in all the three arms compared to the perfect case. The situation becomes completely different for the case of strong disorder i.e., $W=10$. In this case the current in the middle arm almost disappears, while for the other arms
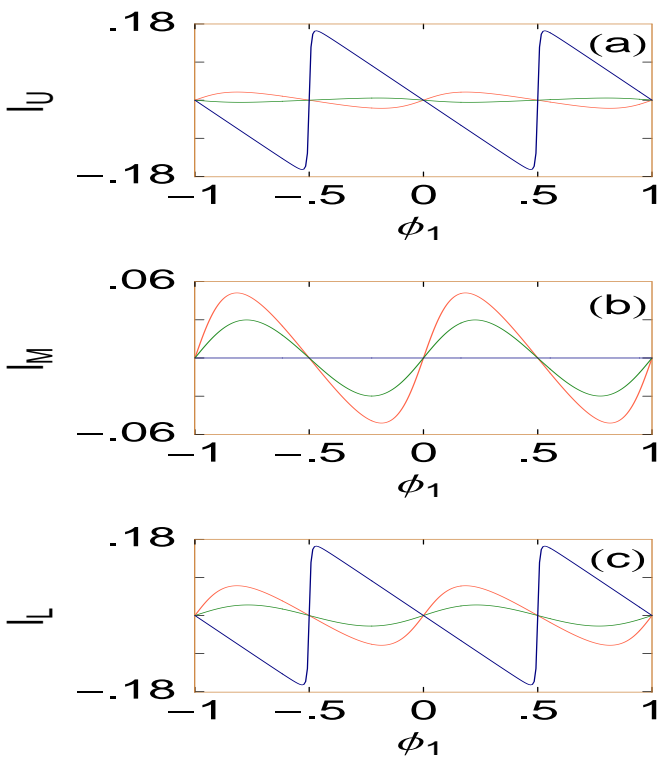

FIG. 5: Current-flux characteristics for the three different arms of a three-arm mesoscopic ring considering $N_{U}=N_{L}=$ 15, $N_{M}=11, \phi_{2}=0.5$ and $N_{e}=15$, where (a), (b) and (c) correspond to the currents in the upper, middle and lower arms, respectively. The red, green and blue curves in each panel represent $W=0,1$ and 10, respectively.

it achieves a high value. Thus a dramatic change in current amplitude takes place for the two different regimes of impurity strength.

To explore this phenomenon more clearly, in Fig. 6. we show the variation of typical current amplitude as a function of $W$, where (a), (b) and (c) give the results for the upper, middle and lower arms, respectively, and (d) corresponds to the variation for the entire ring. The typical current amplitude is evaluated from the relation, $I_{\text {typ }}=\sqrt{\left\langle I^{2}\right\rangle_{\phi_{1}, W}}$, where the averaging is done over a complete period of $\phi_{1}$ and 50 random disordered configurations. From the $I_{\text {typ }}-W$ spectra the effect of disorder is clearly visible. It shows that the current amplitude in the middle arm sharply drops to zero as $W$ is increased. While, for the other two impurity-free arms the current amplitude initially decreases, and reaching to a minimum at a critical value $W=W_{c}$ (say), it again increases with $W$. Thus, beyond the critical disorder strength $W_{c}$, the anomalous behavior is observed and this phenomenon can be implemented as follows. We consider the threearm mesoscopic ring as a coupled system combining two sub-systems, one is ordered and other is disordered. In the absence of any coupling among the ordered and disordered regions, we can think the entire system as a simple 
combination of two independent sub-systems. Therefore, we get all the extended states in the ordered region, while the localized states are obtained in the disordered region. In this situation, the motion of an electron in any one region is not affected by the other. But for the coupled system, the motion of the electron is no more independent, and we have to take the combined effects coming from both the two regions. With the increase of disorder,
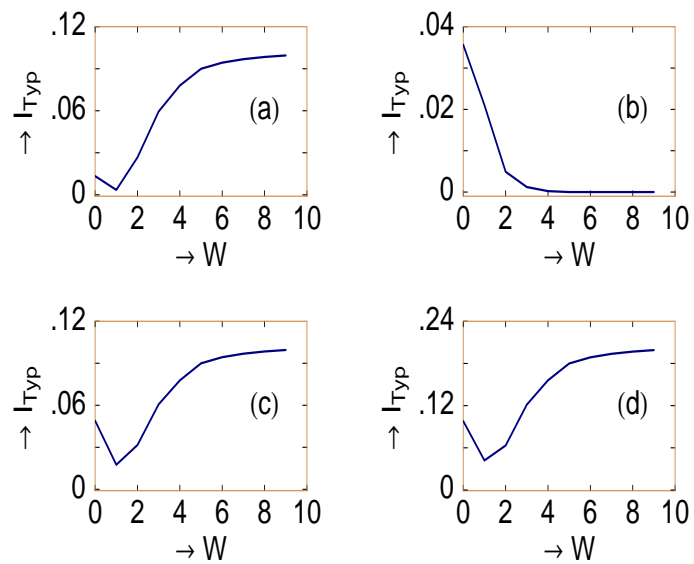

FIG. 6: $I_{\text {typ }}-W$ characteristics for a three-arm mesoscopic ring considering $N_{U}=N_{L}=15, N_{M}=11, \phi_{2}=0.5$ and $N_{e}=15$. (a), (b) and (c) represent the responses for the upper, middle and lower arms, respectively, while (d) corresponds to the variation for the entire ring.

the scattering effect becomes dominated more and thus the reduction of the current is expected. This scattering is due to the existence of the localized eigenstates in the disordered region. Now in the limit of weak disorder the coupling between the two sub-systems becomes strong and the motion of the electron in the ordered region is highly influenced by the disordered region. Therefore, the scattering effect from both the two regions is quite significant and the current amplitude gets reduced. On the other hand, in the strong disorder regime the coupling between the two sub-systems becomes weak and the scattering effect from the ordered region is less significant, and it decreases with $W$. At the critical value $W_{c}$, we get a separation between the much weaker and the strongly localized states. Beyond this value, the weaker localized states become more extended and the strongly localized states become more localized with the increase of $W$. In this situation, the current is obtained mainly from these nearly extended states which provide the larger current with $W$ in the higher disorder regime.

From the above analysis, behavior of the typical current amplitude for the entire system (Fig. 6(d)) can be clearly understood and it shows the similar variation like the upper and lower arms.

\section{CONCLUDING REMARKS}

To summarize, we have explored an idea to investigate the nature of persistent currents in individual branches of a multi-arm mesoscopic ring. Starting with a brief description of persistent current in a traditional singlechannel mesoscopic ring, pierced by an $\mathrm{AB}$ flux $\phi$, we have examined the characteristic features of persistent currents in separate arms of a three-arm mesoscopic ring where the upper and lower sub-rings are subject to $A B$ fluxes $\phi_{1}$ and $\phi_{2}$, respectively. Our analysis may provide a basic framework to address magnetic response in individual branches of any complicated quantum network.

In the present paper we have done all the calculations by ignoring the effects of temperature, electron-electron correlation, electron-phonon interaction, etc. We need further study by incorporating all these effects.

We are thankful to Prof. S. Sil, Prof. B. Bhattacharyya, M. Dey and P. Dutta for useful discussion.
[1] M. Büttiker, Y. Imry, and R. Landauer, Phys. Lett. 96A, 365 (1983).

[2] H. F. Cheung, Y. Gefen, E. K. Riedel, and W. H. Shih, Phys. Rev. B 37, 6050 (1988).

[3] B. L. Altshuler, Y. Gefen, and Y. Imry, Phys. Rev. Lett. 66, 88 (1991).

[4] A. Schmid, Phys. Rev. Lett. 66, 80 (1991).

[5] V. Ambegaokar and U. Eckern, Phys. Rev. Lett. 65, 381 (1990).

[6] S. K. Maiti, Physica E 31, 117 (2006).

[7] S. K. Maiti, Solid State Phenomena 155, 87 (2009).

[8] S. Bellucci and P. Onorato, Physica E 41, 1393 (2009).

[9] P. A. Orellana and M. Pacheco, Phys. Rev. B 71, 235330 (2005).

[10] L. K. Castelano, G.-Q. Hai, B. Partoens, and F. M.
Peeters, Phys. Rev. B 78, 195315 (2008).

[11] L. P. Levy, G. Dolan, J. Dunsmuir, and H. Bouchiat, Phys. Rev. Lett. 64, 2074 (1990).

[12] V. Chandrasekhar, R. A. Webb, M. J. Brady, M. B. Ketchen, W. J. Gallagher, and A. Kleinsasser, Phys. Rev. Lett. 67, 3578 (1991).

[13] E. M. Q. Jariwala, P. Mohanty, M. B. Ketchen, and R. A. Webb, Phys. Rev. Lett. 86, 1594 (2001).

[14] R. Deblock, R. Bel, B. Reulet, H. Bouchiat, and D. Mailly, Phys. Rev. Lett. 89, 206803 (2002).

[15] J. X. Zhong and G. M. Stocks, Nano. Lett. 6, 128 (2006).

[16] C. Y. Yang, J. W. Ding, and N. Xu, Physica B 394, 69 (2007). 